\title{
When Did My Mobile Turn Into A 'Sellphone'? A study of consumer responses to tailored smartphone ads
}

\author{
Nicolette Conti, Charlene Jennett, Jose Maestre and M. Angela Sasse \\ University College London \\ Gower Street, London WC1E 6BT, UK \\ nickyconti@gmail.com, c.jennett@cs.ucl.ac.uk,jomaceld@gmail.com, a.sasse@cs.ucl.ac.uk
}

\begin{abstract}
Tailored push advertising on smartphones is a key target for the advertising industry. We conducted a study with 20 professionals 'in the wild': over 5 consecutive days participants received ads tailored to their personal profiles and geographical location on their personal smartphones. Of the 400 ads sent, participants accepted $20 \%$, rejected $30 \%$, said 'maybe' to $17 \%$. Interviews revealed that accept or reject decisions were driven by specific needs at the time of delivery - e.g. a busy workload. Effective tailoring of smartphone ads requires fine-grained data on users' emotional state and context of use - data that is sensitive and requires significant effort to obtain. Users liked context-relevant ads, but also perceived privacy costs associated with disclosing personal information. To break this conundrum, users need to be able to customize the ad service, e.g. choosing which information to disclose, when to receive ads, what types of ad.
\end{abstract}

Mobile phone. Smartphone. Mobile advertising. Tailored advertising. Marketing.

\section{INTRODUCTION}

The potential to deliver tailored "just-in-time" ads, matched to the recipient's interests and current location, make smartphones a key target for advertisers. In 2011, companies such as Loopt and Telmap announced plans to send location-based coupons to smartphones, suggesting scenarios such as "walk by a Starbucks and get a coupon sent to your phone." But how do consumers feel about this kind of mobile advertising? What factors affect their decision to accept or reject a tailored ad at the time they receive it on their smartphone?

In this paper, we review past literature on mobile advertising (Section 2). Then we describe our in the wild' research study (Section 3) in which we sent tailored smartphone ads to 20 London professionals over a period of 5 consecutive days. Our findings reveal that effective tailoring of smartphone ads requires fine-grained data about the user's context and emotional state - data which is sensitive and effortful to obtain (Section 4). To off-set these privacy costs, we conclude that users should be allowed to customize ad services according to their own personal preferences and we propose 7 customizable dimensions (Section 5).

\section{BACKGROUND}

Several survey studies have investigated consumers' attitudes towards mobile advertising. Perceived utility has been found to be positively related to intention to adopt mobile advertising (Bauer et al., 2005; Merisavo et al., 2007; SoroaKoury and Yang, 2010). Characteristics of the message, such as entertainment, informativeness, credibility, have been found to correlate with perceived advertising value (Hagiran et al., 2005). The inclusion of a discount may also add utility. A recent survey found that $81.9 \%$ of respondents $(N=348)$ were open to receiving mobile coupons on their mobile devices (Wissinger, 2011).

Several survey studies suggest that consumers also have concerns over how their data is collected and used. Tsang et al. (2004) found that consumers generally have negative attitudes towards mobile advertising - unless they have consented to it. Other factors - loss of privacy, irrelevant content and frequency of ads - have also been found to be negatively related to willingness to accept mobile advertising (Haghiran et al., 2005; Merisavo et al., 2007; Wissinger, 2011). 
Surveys are useful for exploring attitudes, but are limited in their ability to predict how participants will react when presented with a tailored ad in a reallife context. To overcome this problem, some researchers have used descriptive scenarios (Kaasinen 2003; Unni and Harmon 2007; Zhang et al. 2010). This involves asking participants to imagine how they would respond to a situation.

Another approach is to run studies 'in the wild', i.e. outside of the lab. These studies are more difficult to design and conduct, but the benefit is that they allow the researcher to have a greater understanding of how people react to novel technology in real world settings. Barwise and Strong (2002) sent SMS ads to 1,000 participants over a period of 6 weeks. When asked what made a good text ad, participants replied 'short and straight to the point' (26\%), 'funny/entertaining' (26\%), 'appeal to area of interest' $(20 \%)$, 'eye catching' (13\%) and 'prize/promotion' (12\%).

Hakkila and Mantyjarvi (2004) gave 9 participants an MMS capable phone and asked them to walk a pre-defined route where they received 6 locationsensitive MMS notifications. Participants categorised notifications as 'own self-set messages', 'message initiated by a friend or personal contact', and 'advertisements'. Ad notifications raised the most concerns because of potential information flooding and spamming.

Hakkila and Isomursu (2005) recruited 20 participants to trial a variety of location-based services over a summer - including an Advertising Tool where they received tailored SMS/MMS ads. Initially participants had high expectations, but they felt frustrated by slow connection times, lack of content and complicated access.

\section{RESEARCH STUDY}

\subsection{Aims}

Previous 'in the wild' studies provide interesting insights into users' experiences; but as of yet none provide a comprehensive account of how a person decides to accept or reject a mobile ad at the time they receive it. There has also been no study focusing on working professionals - arguably an ideal target audience for advertisers as they are familiar with mobile technology and have disposable income.

In our study we aimed to understand what factors influence a person's decision to accept or reject tailored smartphone ads. Over a period of 5 days we sent smartphone ads to 20 London professionals tailored to their interests and location. 10 participants received ads with discounts
(Discount Group) and 10 received ads with product information only (Informative Group). Based on past research (Barwise and Strong, 2002; Wissinger, 2011), we predicted that the Discount Group would respond 'yes' to more ads than the Informative Group.

\subsection{Methodology}

\subsubsection{Participants}

Twenty participants were recruited through an opportunity sample. 11 were female and 9 were male. Their ages ranged from 27 to 41 years, the mean age being 33 years $(S D=4.92)$. All participants were professionals who lived and worked in London. All participants were experienced smartphone users and at the time of the experiment had owned a smartphone for 1 year on average. 15 owned iPhones and 5 owned Androids. All participants had used their smartphone to browse the internet. 16 had experience using mobile location services/apps.

\subsubsection{Materials}

A pre-study questionnaire captured participants' personal interests, pastimes, activities and mostfrequented locations in London.

\subsubsection{Apparatus}

To mimic what smartphone tailored advertising could be like, we adopted a Wizard of Oz approach - developing a prototype which appears to the user to be a functioning system. A web-based desktop system was developed to help wizards - two researchers - create and send ads tailored to participants' interests and location. Ad templates consisted of spaces to insert a title, image, map, textual description, and a question. Using the participant's pre-study questionnaire and their location data (via an in-built Google Maps application), wizards then populated these spaces with the appropriate content. For example, if a participant had ticked 'eating out' in their pre-study questionnaire, expressed a preference for Thai cuisine, and was found to be located in a West London post code, the wizard could find a closely located Thai restaurant using Google maps, and propose the following for lunch: "Enjoy authentic sweet, sour, hot and zingy Thai food @ The Market Thai." The ad would include an image of Thai food, the restaurant's full address, and a Google map image of where the restaurant was located.

Once an ad was created, the system produced a web link which was placed within a text message and sent to the participant. Figure 1 shows an example of an ad sent to the Informative Group. The Discounts Group differed in that they were offered a discounted price, e.g. '50\% off.' 


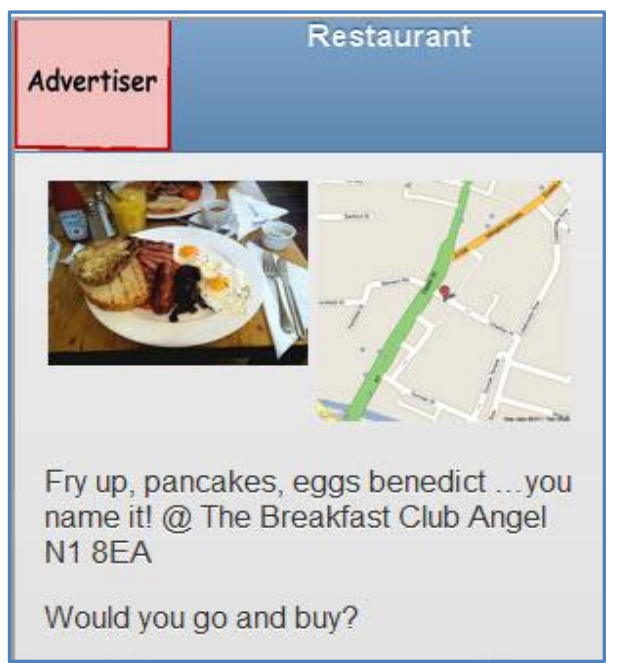

Figure 1: An informative advert

On receiving the text message, participants were expected to click the web link to view the ad and to select a response type - whether they would follow the recommendation ('yes'), ignore the recommendation ('no') or consider it ('maybe'). Participants were then asked to select a reason for their choice. For example, for having chosen 'no', a participant in the Discounts Group could choose one of the following options; 'it was not what I needed', 'it wasn't an attractive discount', 'I found a better discount', 'it was out of my location range.' There was a time limit of 1 hour for viewing and responding to each ad. This was to ensure that participants' responses were situated according to the time of day that the ad was intended.

\subsubsection{Procedure}

Participants were randomly allocated to either the Informative Group or the Discounts Group. Participants filled in a pre-study questionnaire online and were told that they would be trying out a new service ('The Advertiser/Discounter') that automatically collects adverts / discounts from third party retailers, outlets and advertisers and sends tailored matches to their personal smartphone.

Both the Informative and the Discounts groups participated in the experiment within the same 5day period, Weds 6th July to Sun 10th July 2011. Participants were told the start and end date of the experiment, but were not told the number of ads they would receive each day, or the 1 hour time limit for responding to each ad. A total of 20 ads were sent to each participant, 4 ads each day. These were sent during the following intervals: morning (8.30-11.00), lunchtime (12-13.30), afternoon (15-16.30) and evening (17-19.00).

At the end of the experiment, participants were interviewed about their experience and asked to expand on their reasons for 'yes', 'no' and 'maybe' responses. They were also asked to give reasons for when they did not respond to an ad. Each interview lasted app. 15 mins. All participants were fully debriefed and received a £25 gift voucher.

\subsubsection{Qualitative Analysis}

The interview data was analyzed using grounded theory methodology (Strauss \& Corbin, 1990). We used 3 coding procedures: open coding, to identify categories and their properties; axial coding, to understand the relationship between the categories; and selective coding, refining the categories to form a central theory.

\subsection{Results}

\subsubsection{Response Rate}

There were 400 ads sent in total. Participants responded 'yes' to 79 ads (20\%), 'maybe' to 66 ads $(17 \%)$, and 'no' to 120 ads (30\%). 135 ads were not responded to $(34 \%)$.

\subsubsection{Informative Versus Discounts}

Two hundred ads were sent to each group. The Informative Group responded to $143(71.5 \%)$ while the Discounts Group responded to $122(61 \%)$.

Table 1: Frequencies of 'yes', 'maybe' and 'no' responses

\begin{tabular}{|c|c|c|}
\hline Response & $\begin{array}{c}\text { Informative } \\
\text { Group }\end{array}$ & $\begin{array}{c}\text { Discount } \\
\text { Group }\end{array}$ \\
\hline 'Yes' & $41(29 \%)$ & $38(31 \%)$ \\
\hline 'Maybe' & $42(29 \%)$ & $24(20 \%)$ \\
\hline 'No' & $60(42 \%)$ & $60(49 \%)$ \\
\hline Total & $143(100 \%)$ & $122(100 \%)$ \\
\hline
\end{tabular}

Table 1 shows the frequencies of 'yes', 'no' and 'maybe' responses. The majority of ads were rejected in both groups (informative $42 \%$, discount $49 \%$ ). Also there was little difference between the groups in terms of accepting ads: the Discount Group selected 'yes' for $31 \%$ and the Informative Group selected 'yes' for 29\%. A Chi-Square test confirmed that type of advert (informative or discount) did not significantly affect responses, $X^{2}{ }_{(2}$, $\mathrm{N}=265)=3.38, p=.18$.

The most popular reasons selected for 'yes' responses were 'It was useful information' (informative $73 \%$ ) and 'It was a good offer' (discounts 63\%). For 'maybe' responses, the most popular reasons were 'Might have been useful if I needed it' (informative 40\%) and 'I needed more time to think about it' (discounts 33\%). For 'no' responses, the most popular reason was 'It was not what I needed' (informative 47\%, discounts $73 \%$ ).

\subsubsection{Willingness to Sign Up}

The grounded theory (GT) revealed 3 stages that a person must go through before they accept/reject an ad, see Figure 2. There are also several 
barriers, i.e. factors that can stop them from moving from one stage to the next. These stages and barriers will now be described in turn. Quotes from the Discount/Informative Group will be referred to as D/l followed by the participant number, e.g. D1.

The first stage of the GT was concerned with factors that affect whether a participant is willing to sign up to such an advertising service. Participants said that it mattered who was the service provider, what personal information was being used to serve the tailored ads, and how relevant/useful the tailored ads were perceived to be.

Three participants said that they would be willing to sign up if the service provider had a good reputation and was known to be trustworthy. Six participants commented that they were reluctant to share their personal information with "strangers" and felt that tailored ads were akin to being observed by "big brother." Their decision to sign up would depend on whether they were able to control how their personal information was used.

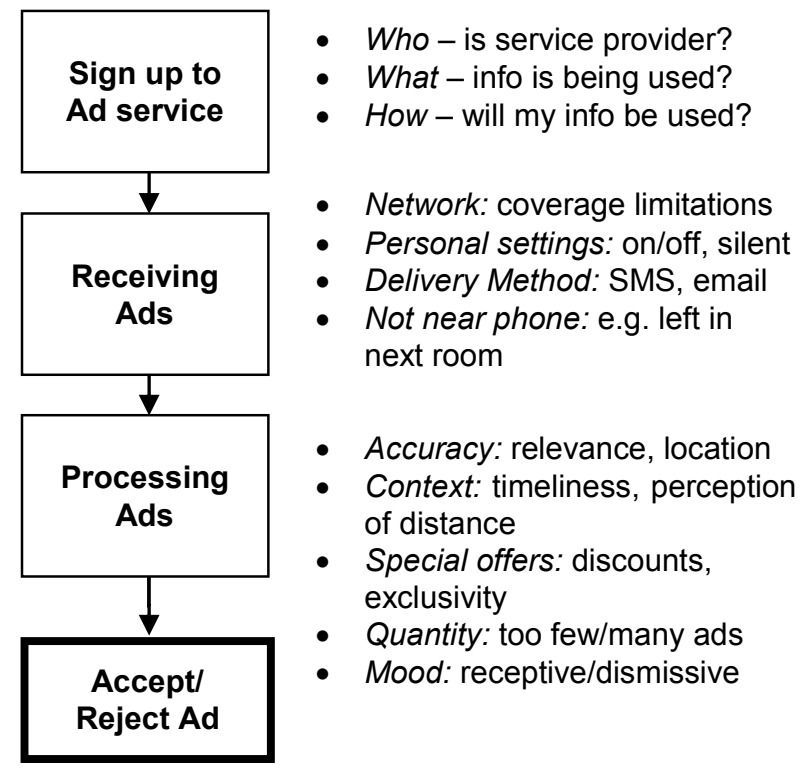

Figure 2: Grounded theory model depicting the process of accepting/rejecting an advert.

\subsubsection{Receiving Adverts}

The second stage of the GT focused on factors that affect whether participants attended to an ad in time. In our study we found that $34 \%$ of ads were not responded to - i.e. within 1 hour of the message being sent. This was confirmed in our interviews 18 participants said that on more than one occasion they were presented with the message 'advert expired' when clicking the link within the text message. Four main factors were identified:

(i) Network limitations: Five participants said that the reason they did not view an ad on time was because of limited or lack of network coverage: "I've got bad reception in the bedroom at my house" (D1); "I would be on my way home on the tube and missed them" (D2).

(ii) Personal phone settings: Four participants said that they purposively chose to turn their phone off or put it on silent during busy time periods, such as when they are in a meeting at work - which meant they missed the ad as a result.

(iii) Delivery method: Four participants suggested that unless the nature of the ad was urgent then they would prefer to receive ads via email. They perceived receiving phone ads as undesirable interruptions, especially during work hours.

(iv) Not near phone: Nine participants said that they missed the ad because they were "not attached" to their phone and had left it unattended (e.g. on their desk or in another room) while they were busy elsewhere.

\subsubsection{Processing Adverts}

The third stage of the GT analysis focused on participants' thoughts when they viewed the ad, and the factors that influenced their decision to accept (respond 'yes') or reject (respond 'no'). Five main factors were identified:

(i) Accuracy: While eight participants expressed surprise at how well an ad fitted their interests/needs, ten participants said that sometimes an ad met their broader interests, but it was not specific/relevant enough to their personal preferences. Accuracy of location targeting was also important. Thirteen participants said that the location "did not always work", i.e. the ad location was not close to where they were positioned at the time.

(ii) Context: Seven participants felt that ads were useful when they arrived at "timely" moments. This was particularly the case during weekday mornings and lunchtimes: "Advertising was happening at good timings[...] when I was thinking of having a meal or going for a coffee" (I3). But there were also missed opportunities. Two participants mentioned losing out because they did not view an ad in time: "The advert asked me 'do you want a coffee?' when I had one in my hand!" (D7). Two participants said that although an ad did appeal to their needs, they could not take advantage of it because they were too busy to leave the office. Twelve 
participants described how ads describing day/evening events appealed to their interests, but they could not make use of them because they already had plans.

The participant's context also affected their perception of distance. E.g. if the ad location was 0.5 miles away this might be considered too far to venture out of the office for coffee/lunch. But outside of work hours, participants did not mind travelling to places further away, particularly if the ad was highly relevant to their interests: "if there is a gig or something then it's natural that you would have to go for it even if it's on the other side of London" (D4).

(iii) Special offers: Seven participants in the Informative Group said that they might have responded more favourably if ads carried discounts. By comparison, participants in the Discounts Group were presented with offers and their opinions varied on a case-to-case basis: "[offers] varied widely from fantastic to not at all" (D7). Three participants described rejecting an ad because it did not offer a big enough discount and/or was not exclusive enough.

(iv) Quantity: Five participants were surprised that the experience of receiving ads was not as irritating as they originally thought. Seven participants thought the amount of ads sent each day was adequate. Seven participants felt they received too many ads- they found it particularly irritating when they were busy and incoming ads would "break their trail of thought."

(v) Mood: Some participants were more receptive to ads than others. Three participants said that they preferred to search for items themselves and disliked push ads. Two participants described how sometimes an ad appealed to their interests/needs, but they were "not in the mood" to act upon it. Three participants described how the experience of receiving ads could be disappointing: "When I get a text message it is from someone I know or a friend and not adverts so when I see it's an ad I'm kind of disappointed" (14).

\section{DISCUSSION}

In our study we aimed to understand participants' reasons for accepting or rejecting smartphone tailored ads. Our hypothesis regarding the two groups was not supported: the Discount Group did not respond 'yes' to significantly more ads than the
Informative Group. We suggest that although discounts are important (Wissinger, 2011), it is only one of a number of factors that influence whether a person accepts an ad or not.

Our GT analysis revealed that there are three stages participants go through (see Figure 2). First they must decide whether they are willing to sign up to such an ad service. Privacy concerns such as 'how will my info be used?' will affect this decision. In support of Adams and Sasse's privacy model (2001), this suggests that trust is a key determinant of information disclosure.

Next, when sent a tailored ad, the recipient needs to attend to it in a timely manner. $34 \%$ of ads were not responded to within the time limit. Lack of network coverage was one of the reasons participants gave for not responding to an ad in time. In metropolitan areas, a user often cannot get coverage in buildings, in the shadow of buildings, tunnels etc. even if there is a good network. This is a key factor that may interfere with advertisers' ability to target ads as specifically as users expect. One possible way to overcome this is to have seamless coverage by making wider automatic use of wifi networks - however this would have major implications for mobile providers' business models because they would need to agree to provide seamless wifi access free of charge.

Another reason for not responding to an ad was "being stuck in meetings." All of our participants were working professionals, employed full-time in busy office environments. This meant that they had the disposable income to afford ad offers - but at the same time, they might also have less free time to take advantage of these offers compared to other user groups, e.g. students. This suggests that the type of target consumer and context in which ads are received are important factors to take into account for any push mobile advertising service.

Finally, the recipient must process the ad content and make a decision about whether to accept or reject. Past research suggests that the more relevant the ad is, the higher the perceived usefulness (Zhang et al., 2010). However our results suggest the situation is more complex. During work hours, a recipient might be more likely to view ads as an annoyance and might prefer close locations. During leisure periods, the same recipient might be more willing to receive a higher quantity of ads, advertising for products/events not in their immediate location. Again this emphasizes the importance of fully understanding the context of the recipient. There are also other factors not related to ad content which can affect a person's decision, e.g. the recipient might have already made plans, or might just be "not in the mood". 


\section{CONCLUSION}

Our research captured the reactions of working professionals experiencing smartphone tailored ads in the context of their everyday lives. The results suggest that there are barriers that advertisers must overcome if smartphone tailored advertising is to be successful. Effective tailoring requires finegrained data on users' emotional states and contexts of use- but at the same time, the amount of information needed to provide more relevant ads is perceived as invasive. To break this conundrum, consumers must be given choice and the ability to customize either through their own smartphone or via a personalized online application.

We identify several factors from our study which could be used as customizable dimensions:

- Service availability - ability to turn on/off.

- Delivery method - ability to switch between mobile and email notifications.

- Time - selecting periods to receive ads.

- Personal interests - choose, add, edit or remove personal preferences.

- Location - limiting/extending distance of advertised items away from current personal location in minutes/miles.

- Ad preferences - choose between offers, information, sales etc.

- Quantity - ability to specify number of ads received during any given time period.

At the same time advertisers will need to find a way of offering customization that does not require too much effort on the user's part. Past research suggests that users do not want to spend long periods of time populating profiles and giving feedback to recommender systems (Bonhard and Sasse, 2006). Advertisers might also consider the use of persuasion/rewards to motivate users.

In future research it will be important to explore the generalizability of our findings by replicating our study with a larger sample and a diverse range of smartphone users. It will also be useful to explore how a person's reactions to an advertising service change over a longer period of time and whether there are any novelty/habituation effects.

\section{ACKNOWLEDGMENTS}

We would like to thank Ashweeni Beeharee and Sven Laqua for their support.

\section{REFERENCES}

Adams, A. and Sasse, M. A. (2001). Privacy in multimedia communications: Protecting users, not just data. In Blandford and Vanderdonckt (Eds.),
People and Computers XV- Interaction without frontiers, 49-64. Springer: London.

Barwise, P. and Strong, C. (2002). Permissionbased mobile advertising. Journal of Interactive Marketing 16 (1), 14-24.

Bauer., H. H., Reichardt, T., Barnes, S. J. and Neumann, M. M. (2005). Driving consumer acceptance of mobile marketing: A theoretical framework and empirical study. Journal of Electronic Commerce Research 6 (3), 181-192.

Bonhard, P. and Sasse, M. A. (2006). I thought it was terrible and everyone else loved it: A new perspective for effective recommender system design. In McEwan (Ed.), People and Computers XIX-The Bigger Picture, 251-265. Springer: London Kaasinen, E. (2003). User needs for location-aware mobile services. Personal and Ubiquitous Computing 7 (1), 70-79.

Haghiran, P., Madlberger, M. and Tanuskova, A. (2005). Increasing advertising value of mobile marketing- An empirical study of antecedents. Proc. of $28^{\text {th }}$ Hawaii International Conference on System Science 2005, IEEE.

Hakkila, J. and Isomurso, M. (2005). User experiences on location-aware mobile services. Proc. of OZCHI 2005.

Hakkila, J. and Mantyjarvi, J. (2004). User experiences on combining location sensitive mobile phone applications and multimedia messaging. Journal of Mobile Multimedia 1 (1), Rinton Press.

Merisavo, M., Kajalo, S., Kajaluoto, H., Virtanen, V, Salmenkivi, S., Raulas, M. and Leppaniemi, M. (2007). An empirical study of the drivers of consumer acceptance of mobile advertising. Journal of Interactive Advertising 7 (2), 41-50.

Soroa-Koury, S. and Yang, K. C. C. (2010). Factors affecting consumers' responses to mobile advertising from a social norm theoretical perspective. Telematics \& Informatics, 27, 103-113. Strauss, A. and Corbin, J. (1990). Basics of qualitative research: Grounded theory procedures and techniques. Sage: London.

Tsang, M. M., Ho, S.-C. and Liang, T.-P. (2004). Consumer attitudes toward mobile advertising: An empirical study. International Journal of Electronic Commerce, 8 (3), 65-78.

Unni, R. and Harmon, R. (2007). Perceived effectiveness of push vs. pull mobile location-based advertising. Journal of Interactive Advertising 7 (2), 28-40.

Wissinger, C. (2011). Mobile users have mixed feelings about location-based coupons, according to latest mobile survey. http://www.prweb.com/releases/ProsperMobileSep 11/Coupons/prweb8851191.htm (retrieved 13 Aug, 2012).

Zhang, H., Guerrero, C., Wheatley, D. and Lee, Y. S. (2010). Privacy issues and user attitudes towards targeted advertising: A focus group study. Proc. of Human Factors and Ergonomics Society Annual Meeting 54 (19), 1416-1420. 